\title{
Pheromone Exposure Influences Preoptic Arginine Vasotocin Gene Expression and Inhibits Social Approach Behavior in Response to Rivals but Not Potential Mates
}

\author{
Lisa A. Mangiamele ${ }^{a}$ b Alex D.T. Keeney ${ }^{b}$ Erin N. D'Agostino ${ }^{b}$ \\ Richmond R. Thompson ${ }^{a, b}$ \\ ${ }^{a}$ Department of Psychology and ${ }^{b}$ Program in Neuroscience, Bowdoin College, Brunswick, Me., USA
}

\section{Key Words}

Androstenedione · Arginine vasotocin · Parvocellular preoptic area $\cdot$ Social behavior $\cdot$ Teleost

\begin{abstract}
The nonapeptides arginine vasotocin (AVT) and vasopressin mediate a variety of social behaviors in vertebrates. However, the effects of these peptides on behavior can vary considerably both between and within species. AVT, in particular, stimulates aggressive and courtship responses typical of dominant males in several species, although it can also inhibit social interactions in some cases. Such differential effects may depend upon AVT influences within brain circuits that differ among species or between males that adopt alternative reproductive phenotypes and/or upon the differential activation of those circuits in different social contexts. However, to date, very little is known about how social stimuli that promote alternative behavioral responses influence AVT circuits within the brain. To address this issue, we exposed adult male goldfish to androstenedione (AD), a pheromonal signal that is released by both males and females during the breeding season, and measured social approach responses of males towards same- and other-sex individuals before and after $A D$ exposure. In a second experiment, we
\end{abstract}

measured AD-induced AVT gene expression using in situ hybridization. We found that brief exposure to AD induces social avoidance in response to rival males, but does not affect the level of sociality exhibited in response to sexually receptive females. Exposure to AD also increases AVT gene expression in the preoptic area of male goldfish, particularly in the parvocellular population of the preoptic nucleus. Together, these data suggest that $A D$ is part of a social signaling system that induces social withdrawal specifically during male-male interactions by activating AVT neurons.

Copyright $\odot 2013$ S. Karger AG, Basel

\section{Introduction}

Arginine vasotocin (AVT) and its mammalian homologue, arginine vasopressin (AVP), are nonapeptides that play important roles in the regulation of social behavior in diverse vertebrate groups. For example, AVT/AVP stimulates sexual behavior in amphibians [Moore and Miller, 1983; Marler et al., 1995], influences social bonding in mammals [Winslow et al., 1993; Wang et al., 1994], and is associated with aggression and social dominance in fish, birds, and mammals [Goodson and Bass, 2001; Goodson, 2008; reviewed in Albers, 2012; Godwin and

\section{KARGER}

E-Mail karger@karger.com

www.karger.com/bbe
(C) 2013 S. Karger AG, Basel

0006-8977/13/0813-0194\$38.00/0
Lisa A. Mangiamele

Department of Psychology, Bowdoin College 6900 College Station

Brunswick, ME 04011-8469 (USA)

E-Mail lmangiam@bowdoin.edu 
Thompson, 2012]. These diverse behavioral effects are mediated primarily by forebrain AVT/AVP cell groups, the most conserved of which are located in the preoptic area and hypothalamus, with projection patterns that extend out to the pituitary, forebrain, and hindbrain [reviewed in Goodson and Bass, 2001; Goodson and Thompson, 2010]. In many species, social stimuli activate AVT/ AVP cells and are associated with the subsequent release of these peptides in the brain. For example, positive social stimuli (e.g., contact with a sexual partner or same-sex bird) activate AVT cells in the medial bed nucleus of the stria terminalis of males in several gregarious bird species [Goodson and Wang, 2006; Goodson, 2008], and the subsequent release of AVT from those cells into the septum mediates behavioral preferences for large groups [Kelly et al., 2011]. However, the behavioral effects of AVT/AVP can vary considerably depending on the context of the social signal. In male prairie voles (Microtus ochrogaster), AVP release in the anterior hypothalamus is higher in response to social contact with a male or female stranger, compared to contact with a pair bond partner, and is associated with selective aggression towards unfamiliar individuals. Yet, these males still maintain a high level of social affiliation with their female partners [Gobrogge et al., 2007]. Thus, social cues that drive endogenous AVT/ AVP release in neural circuits underlying behavior are likely important factors that facilitate appropriate responses to the social environment, including the display of both prosocial and antisocial behaviors.

Endogenous AVT/AVP production and release from different neuronal populations could explain some of its differential effects on behavior. Certain social signals may trigger AVT/AVP release in some behavioral circuits but not others, thus facilitating responses that are context specific and even exerting the opposite effect on the same behavior in different contexts. In fact, positively and negatively valenced social stimuli activate different populations of AVT/AVP cells in zebra finches [Goodson and Wang, 2006] and mice [Ho et al., 2010], and in several avian species endogenous AVT facilitates aggression in the context of mate competition, but either reduces it or has no effect in other social contexts [Goodson et al., 2009; Kabelik et al., 2009]. These studies indicate that it is critical to learn more about how social stimuli that promote alternative behavioral responses influence AVT circuits in the brain.

Goldfish (Carassius auratus) are a good model in which to further investigate the social stimuli that generate context-dependent AVT effects. Goldfish are a highly social species that congregate in mixed-sex groups [Ma-

AVT Responses to Pheromone Exposure gurran and Pitcher, 1983; Kavaliers, 1989]. During the breeding season, males engage in a 'scramble competition' for mates, in which many individuals compete for access to a single female [Taborsky, 2001]; however, males rarely engage in overt forms of aggression. Instead, they use 'chasing' and 'pushing' behaviors to ensure contact with ovulating females and to outcompete rival males. In these animals, AVT influences the simple approach responses that may underlie these behaviors. Intracranial administration of AVT in male goldfish inhibits the tendency to approach conspecifics [Thompson and Walton, 2004] through its effects on the hindbrain [Thompson et al., 2008b; Thompson and Walton, 2009]. In some social situations, a reduced tendency to approach a conspecific could be advantageous: for example, withdrawing from a rival male if the probability of winning a competition is low. Yet, in other cases, it could be disadvantageous. Failing to chase females when they are ready to release eggs would decrease a male's probability of reproduction. This raises the critical question, what factors regulate AVTinduced social avoidance in goldfish in a way that generates the appropriate behavioral response for a given social situation?

One possibility is that the production of AVT is regulated by a social signal that promotes social withdrawal. Goldfish use a well-characterized suite of chemical signals to communicate socially relevant information, which are known to affect the behavior and physiology of males and females [reviewed in Stacey et al., 2003]. One of the major steroidal pheromones produced by both sexes is androstenedione $(\mathrm{AD})$. Females release a pheromone mixture that contains $\mathrm{AD}$ approximately $12 \mathrm{~h}$ prior to ovulation, long before they are ready to spawn, and the levels of $\mathrm{AD}$ subsequently decline as ovulation approaches [Scott and Sorensen, 1994], whereas sexually active males release comparatively larger quantities of $\mathrm{AD}$, particularly when paired with male and/or female conspecifics [Sorensen et al., 2005]. Such social stimuli could activate AVT cells or drive peptide release in specific neural circuits that mediate appropriate behavioral responses. Thus, AVT-induced social avoidance could play an important role in coordinating male-female interactions to coincide with ovulation or in mediating male-male competitive interactions.

In this study, we asked how AD influences male sociality within a reproductive context. To do this, we exposed males to $\mathrm{AD}$ or a control solution and tested their willingness to approach other males (potential rivals) and females (potential mates) in a social approach paradigm. To isolate the effects of the pheromonal signal, we used a 
visual approach paradigm, in which males were prevented from physical or chemical contact with other individuals but could interact socially across a Plexiglas barrier. We have previously demonstrated that goldfish can make sexual discriminations using only visual cues [Thompson et al., 2004], although males and females do not obviously differ in appearance to the human eye. We also asked whether AD influences the production of AVT in neural circuits that are likely involved in generating these approach/avoidance responses. Specifically, we measured AVT gene expression in the parvocellular, magnocellular, and gigantocellular populations of the preoptic area. In other teleosts, AVT in the different preoptic nuclei is associated with distinct social responses; levels of AVT mRNA and protein expression in the parvocellular portion of the preoptic area correlate strongly with the tendency to flee from a social encounter, whereas increased AVT in the magno- and gigantocellular portions is associated with increased aggression and courtship behaviors [Semsar and Godwin, 2003; Greenwood et al., 2008; Dewan and Tricas, 2011]. Thus, if AD regulates the influences of AVT on approach behavior in goldfish in a social context-dependent manner, we predicted that the effects of $\mathrm{AD}$ should differ in different preoptic regions, or that AD would induce AVT only in preoptic nuclei associated with social avoidance.

\section{Materials and Methods}

\section{Animals}

Adult male comet goldfish (12-15 cm length) were purchased from Hunting Creek Fisheries (Thurmont, Md., USA) or Mount Parnell Fisheries (Mercersberg, Pa., USA) and housed in the laboratory in 200-gallon stock aquaria with $15-50$ other males. They were fed once per day with pellet food. We held fish in long daylight conditions ( $14 \mathrm{~h}$ light: $10 \mathrm{~h}$ dark) at $20^{\circ} \mathrm{C}$ during the early spring and summer breeding season and in shorter daylight conditions ( $12 \mathrm{~h}$ light: $12 \mathrm{~h}$ dark) at $18^{\circ} \mathrm{C}$ during fall and winter. In all experiments, we confirmed that males were in reproductive condition by noting the expression of milt upon application of light pressure to the abdomen. All animal procedures were approved by the Research Oversight Committee (Institutional Animal Care and Use Committee) at Bowdoin College.

\section{Behavioral Testing}

To determine the effect of $\mathrm{AD}$ on behavior, we used a withinsubject design to measure social approach behavior in male goldfish in response to male $(n=20)$ and female conspecifics $(n=16)$. We tested each fish on 2 consecutive days following the procedure described by Thompson and Walton [2004]. The testing tank consisted of a central 70-liter experimental chamber $(25 \mathrm{~cm}$ long $\times 42$ $\mathrm{cm}$ wide $\times 35.5 \mathrm{~cm}$ deep) bordered on each side by an 18 -liter chamber $(16.5 \mathrm{~cm}$ long $\times 42 \mathrm{~cm}$ wide $\times 35.5 \mathrm{~cm}$ deep $)$ used to hold stimulus fish. Clear Plexiglas partitions between the chambers allowed animals to see each other but prevented chemical exchange between chambers. These partitions allowed UV wavelengths as low as $300 \mathrm{~nm}$ to pass through, and full-spectrum lights (Reptisun 10.0 UVB, Zoo Med Laboratories, San Luis Obispo, Calif., USA) were hung above the tank. We videotaped the behavior of males in the experimental chamber using monochrome cameras (Limelight Video Tracking System, Actimetrics, Wilmette, Ill., USA).

On each day of testing, we allowed the subject fish to acclimate for $15 \mathrm{~min}$ in the experimental chamber before testing. Following the acclimation period, we videotaped the male's behavior for 15 min and recorded the amount of time the fish spent with its nose within 1 in of each Plexiglas partition in order to determine initial side preference. We then placed either an unfamiliar male or female fish into the stimulus chamber opposite the side in which the subject male spent more time in proximity. The stimulus fish was chosen at random from a single-sex group tank on both days of testing. With the stimulus fish in the chamber, we used a peristaltic pump to deliver a solution containing either $\mathrm{AD}$ dissolved in 95\% methanol (final concentration: $1 \times 10^{-7} \mathrm{M}$; Sigma Aldrich, St. Louis, Mo., USA) or methanol vehicle to the experimental chamber on the side containing the stimulus fish at a rate of $10 \mathrm{ml} / \mathrm{min}$. The outlet of the tube delivering the solution was positioned just below the water surface in the center of the Plexiglas partition. We chose the dose of $\mathrm{AD}$ based on the concentration that has been demonstrated to elicit the greatest response from the olfactory epithelium of male goldfish [Sorensen et al., 2005]. Flow rates were adapted from Poling et al. [2001]. We again recorded the behavior of the subject fish for $15 \mathrm{~min}$. We defined social approach behavior as the time the subject fish spent in proximity to the stimulus fish minus the time spent in proximity to that same side during the initial side preference test. The order of exposure to $\mathrm{AD}$ or vehicle solution was counterbalanced across the 2 days of testing.

\section{Pheromone Exposure for Brain Collection}

Because we used a counterbalanced within-subject design for our behavioral experiments, brains could not be collected immediately after AD exposure. Thus, we conducted a second experiment to assess whether AD influences AVT expression in the brain. In that experiment, male goldfish were housed individually in 10-liter tanks at $20^{\circ} \mathrm{C}$ in a dark room overnight. Before the lighton period (7:00 a.m.), we exposed half of the fish $(\mathrm{n}=8)$ to a $1-\mathrm{ml}$ bolus of $\mathrm{AD}$ in $95 \%$ methanol in the tank water (final concentration: $\left.1 \times 10^{-7} \mathrm{M}\right)$ while the other half $(\mathrm{n}=8)$ were exposed to an equivalent volume of methanol vehicle for $1 \mathrm{~h}$. The time of day was chosen to coincide with the natural spawning cycle, which occurs just prior to dawn. After pheromone exposure, fish were immediately anesthetized by immersion in tricaine methanesulfonate (MS-222; Sigma Aldrich) and rapidly decapitated.

\section{Tissue Preparation and AVT mRNA in situ Hybridization}

We embedded brains in M-1 embedding medium (Thermo Scientific, Kalamazoo, Mich., USA), rapidly froze them on dry ice, sectioned them on a cryostat at $20 \mu \mathrm{m}$ in four series, and mounted them on Superfrost Plus slides (Fisher Scientific, Pittsburg, Pa., USA). We generated radioactively $\left({ }^{35} \mathrm{~S}\right)$ labeled sense and antisense riboprobes by reverse transcription from a cDNA template of $C$. auratus AVT using a MAXIscript kit (Ambion, Austin, Tex., USA). The 439-bp template [GenBank Accession No. JX982125], representing the $3^{\prime}$ end of $C$. auratus AVT, was generated by PCR 
Fig. 1. Low $(\times 4)$ and high $(\times 40)$ magnification photomicrographs of AVT-expressing cells sampled in the parvocellular (a, d), magnocellular (b, e), and gigantocellular (c, f) nuclei of the preoptic area. Boxes indicate areas depicted in high magnification photos. f Arrowheads point to gigantocellular cells. a-c Bar $=200 \mu \mathrm{m}$. $\mathbf{d - f}$ Bar $=40$ $\mu \mathrm{m}$.
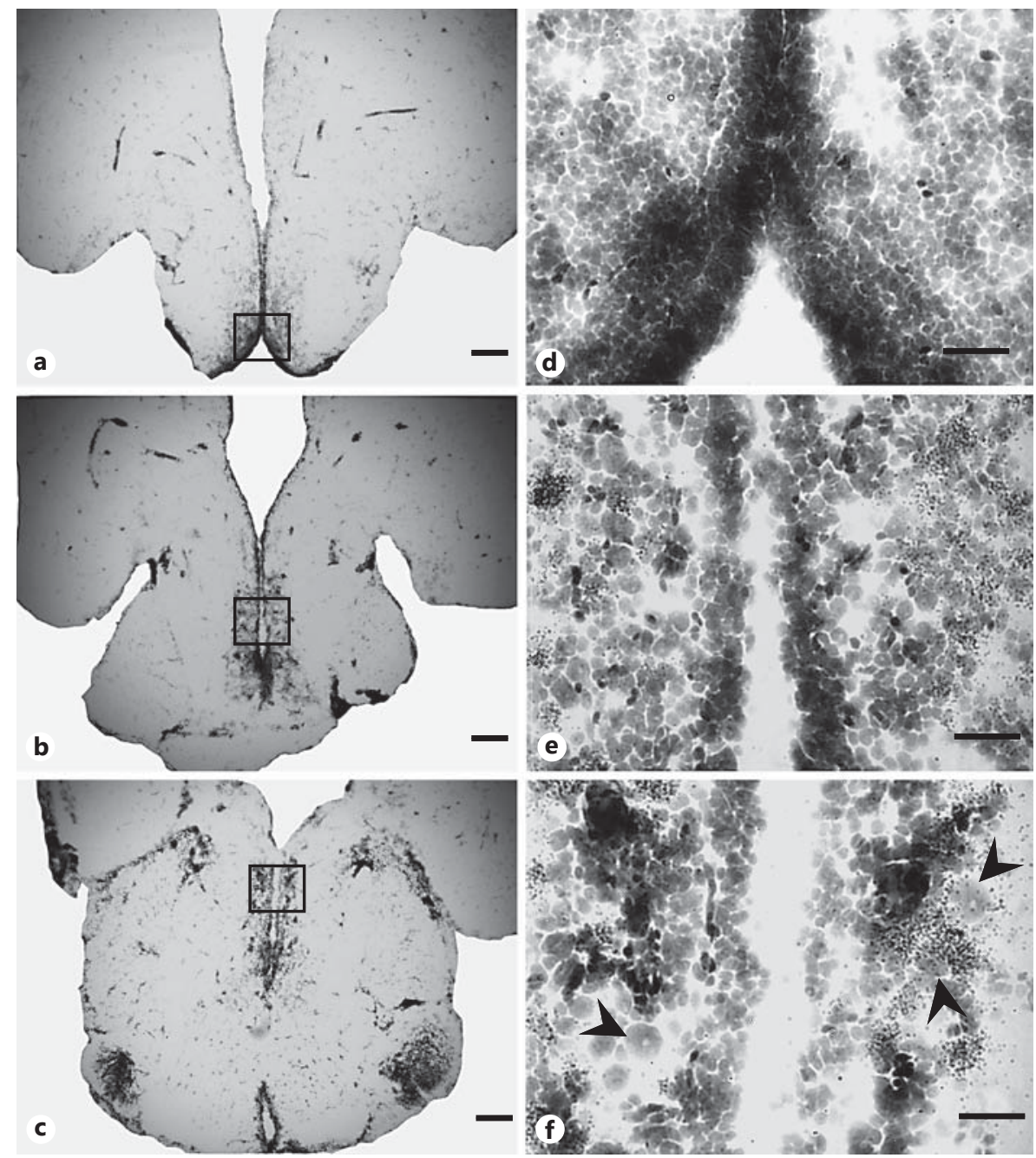

amplification using a 3' RACE system (Life Technologies, Grand Island, N.Y., USA) with the following primers: a gene-specific forward primer ( $5^{\prime}$-CAGACAGTGTCCGGCGTGTGGT- $3^{\prime}$ ) and a universal amplification reverse primer (5'-GGCCACGCGTCGACTAGTAC- $\left.3^{\prime}\right)$. We performed in situ hybridization according to the protocol described by Greenwood et al. [2008]. To visualize the bound riboprobe, we dipped slides in Kodak NTB autoradiography emulsion, allowed them to dry overnight, and stored them in lightproof boxes at $4^{\circ} \mathrm{C}$ for $6 \mathrm{~h}$. Slides were then developed and counterstained with cresyl violet. We confirmed the specificity of our riboprobe for AVT by noting the absence of binding (i.e., silver grains over tissue sections) on slides treated with sense probe.

\section{Quantification of AVT Expression}

We measured AVT expression in three anatomically distinct regions of the preoptic area: the parvocellular, magnocellular, and gigantocellular nuclei (fig. 1), as in Greenwood et al. [2008]. Because the parvocellular and magnocellular nuclei partially overlap in their rostral-caudal extent, we restricted our measurements to the anterior portion of the parvocellular nucleus and the posterior portion of the magnocellular nucleus. For the parvocellular nucle- us, we started sampling at the first evidence of expression in the ventral-most part of the preoptic area and stopped sampling when magnocellular cells (approx. $2 \times$ the size of parvocellular cells) began to appear. For the magnocellular nucleus, we started sampling when the entopeduncular nucleus first appeared on a section and continued for four consecutive sections. For the gigantocellular nucleus, we started at the most caudal section that contained gigantocellular AVT expression and continued rostrally until this nucleus disappeared. The three nuclei each contain cell populations that are easily distinguished by their soma size (parvocellular $<$ magnocellular < gigantocellular); however, our sampling of magnocellular and gigantocellular nuclei often included more than one cell type (fig. 1d-f). For all nuclei, we collected images for a minimum of three consecutive sections and on both hemispheres of the brain where possible.

We quantified AVT expression from digital photomicrographs taken with $\mathrm{a} \times 100$ objective and a Q Imaging Qicam camera. We used a blue optical filter to minimize the appearance of cresyl violet-stained cells so that only silver grains (i.e., AVT expression) appeared in the final image. For each section sampled, we took two images: a blue-filtered image showing silver grains in the preoptic 
Fig. 2. AD influences social investigation of conspecific males but not females. a Social approach responses of males towards other males when exposed to AD or control solution. b Social approach responses of males towards females when exposed to $\mathrm{AD}$ or control solution. In both graphs, social approach score represents the time spent in proximity to the stimulus fish minus the time spent in proximity to that same side of the tank during an initial side preference test. Bars represent mean social approach score \pm SEM. ${ }^{*} \mathrm{p}<0.05$.

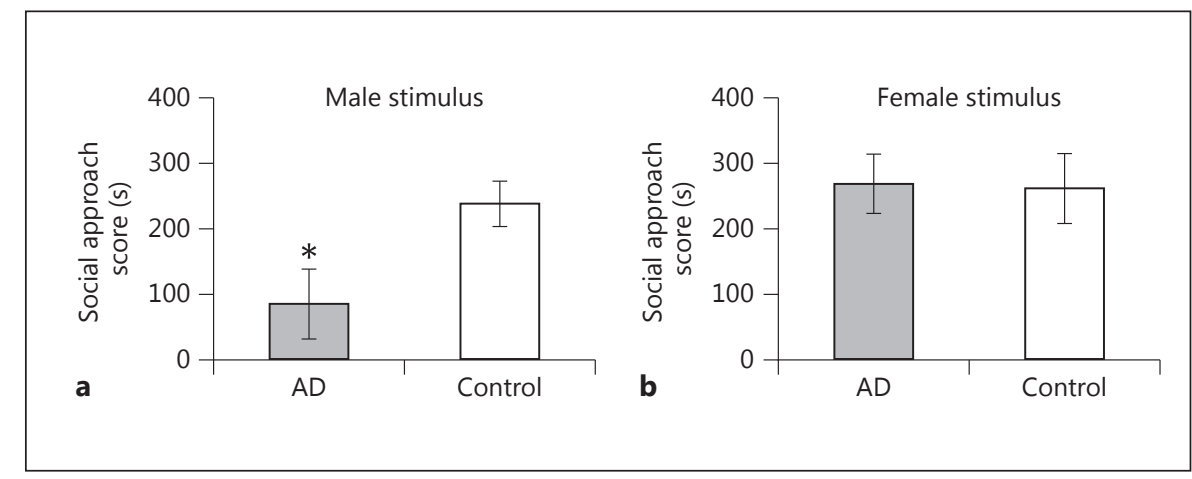

area of interest and a blue-filtered image of an area of the slide containing no tissue to represent local background silver grain density. Brightness and contrast settings were the same for each photo of a given section. We used ImageJ (National Institutes of Health, Bethesda, Md., USA) to convert the images to binary and to measure the area covered by silver grains in each image in pixels ${ }^{2}$. Our final measure of AVT mRNA expression for each section was the area covered by silver grains above background. For each brain region, we calculated mean AVT expression from all images taken for an individual fish.

\section{Statistical Analysis}

To test for an effect of AD on social approach behavior towards male or female conspecifics, we used paired t tests to compare social approach scores in response to AD and control solutions. To determine whether pheromone exposure influenced AVT expression in the preoptic area of males, we used a mixed linear model (ANOVA) with brain region (parvo-, magno-, or gigantocellular nucleus), fish ID, treatment (AD or methanol control), and treatment $\times$ brain region as factors. Fish ID was modeled as a random factor. Brain region was nested within fish ID to account for the fact that AVT expression in different preoptic nuclei from the same brain may be correlated. We followed up our ANOVA with planned post hoc $t$ tests to compare the level of AVT expression in response to $\mathrm{AD}$ and control-stimulated fish in each anatomically distinct preoptic nucleus individually. We did this because the AVT cells in the three preoptic nuclei are morphologically distinct and may be part of different neural circuits [Godwin and Thompson, 2012] and because recent work in teleosts has demonstrated that AVT mRNA and protein expression are associated with different social responses [Greenwood et al., 2008; Dewan and Tricas, 2011]. Thus, we were interested in testing the effects of AD separately on each cell population to test the hypothesis that $\mathrm{AD}$ would induce AVT in some preoptic nuclei but not others. We used JMP 9.0 (SAS, Cary, N.C., USA) for all statistical analyses.

\section{Results}

We found that $\mathrm{AD}$ exposure decreased social approach behavior of males in response to male conspecifics $\left[\mathrm{t}_{(19)}=\right.$ 2.27, $\mathrm{p}=0.03$; fig. $2 \mathrm{a}$ ] but did not affect the behavior of males towards females $\left[\mathrm{t}_{(15)}=0.11, \mathrm{p}=0.91\right.$; fig. $2 \mathrm{~b}$ ].

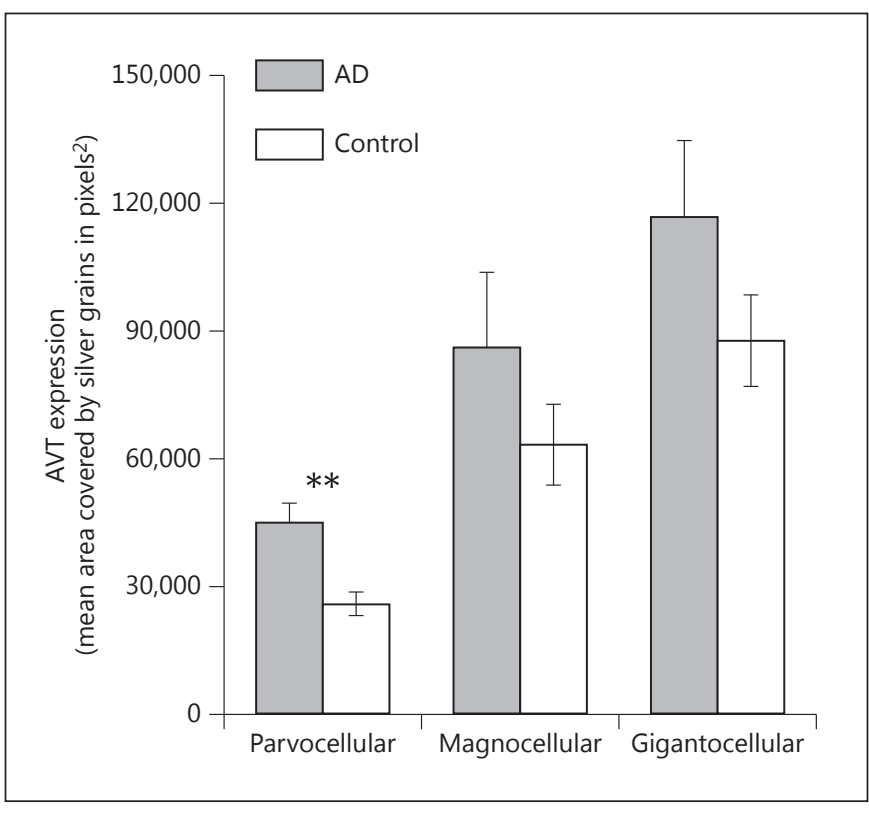

Fig. 3. AVT mRNA levels in the parvocellular, magnocellular, and gigantocellular portions of the preoptic area of males exposed to AD or control solution. Data are graphed as mean area covered by silver grains \pm SEM. ${ }^{* *} \mathrm{p}<0.01$ for planned post hoc comparisons.

When exposed to $\mathrm{AD}$, males spent an average of 87.5 \pm 52.5 s approaching other males, compared to $238.7 \pm$ $33.9 \mathrm{~s}$ for the same males when they were exposed to a control solution. Our recording cameras could not simultaneously collect proximity and activity data, but qualitative observations of males exposed to AD suggested that they were avoiding social contact, as opposed to simply becoming less active. Other studies have also not noted changes in general activity in response to $\mathrm{AD}$ exposure [Poling et al., 2001] or AVT administration [Thompson and Walton, 2004]. 


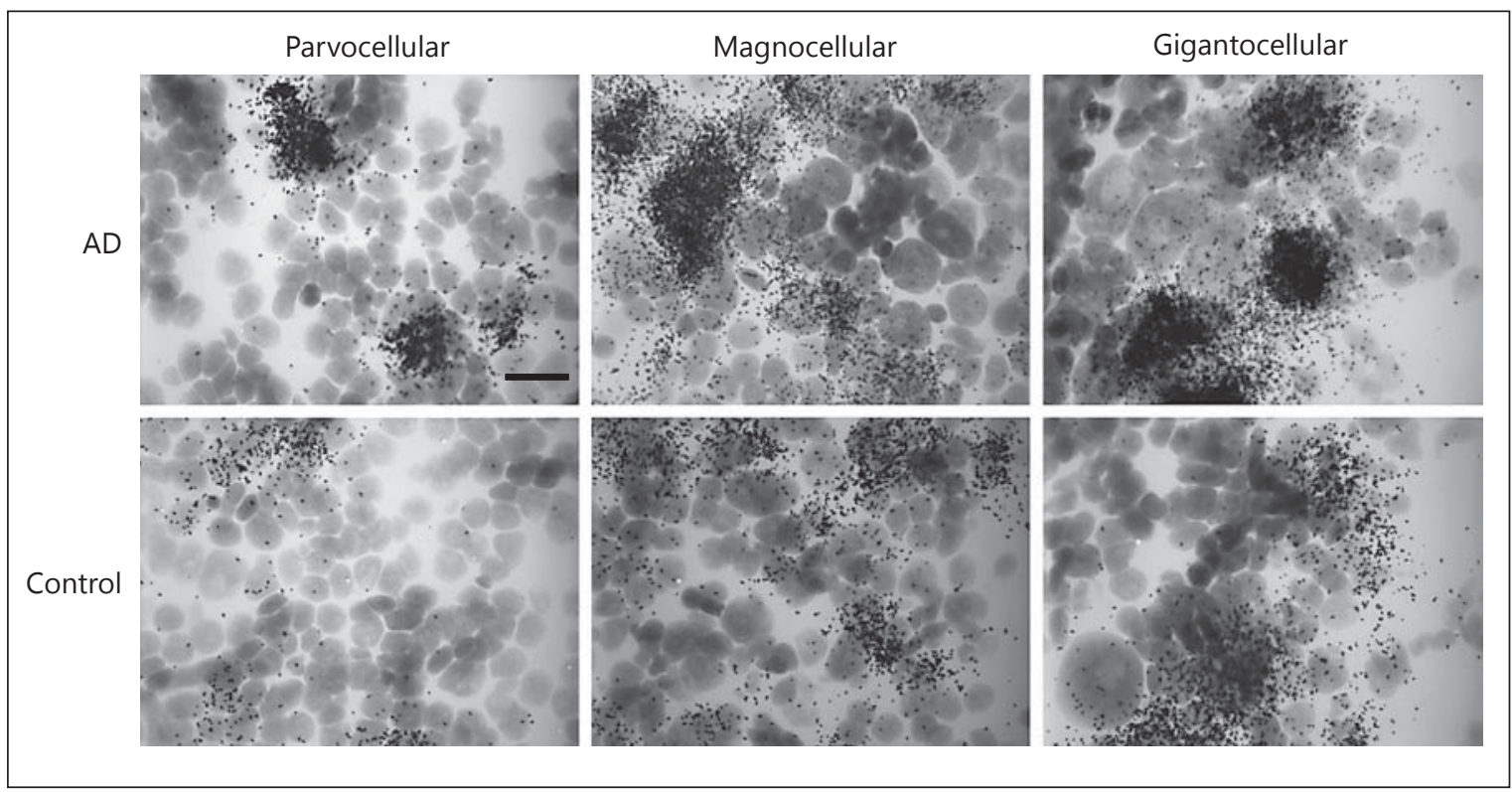

Fig. 4. Representative photomicrographs showing AVT mRNA expression in response to AD or control solution in the parvocellular, magnocellular, and gigantocellular nuclei of the preoptic area. AVT expression is represented by the area within the field of view covered by black spots (silver grains). Cell bodies were counter-stained with cresyl violet. Scale bar $=20 \mu \mathrm{m}$.

We found that $\mathrm{AD}$ exposure also increases overall AVT expression in the male brain when compared to controls [treatment, $\mathrm{F}_{(1,12)}=7.96, \mathrm{p}=0.01$ ]. There was not a significant treatment-brain region interaction $\left[\mathrm{F}_{(2,12)}=\right.$ $1.69, \mathrm{p}=0.22]$; however, when we performed planned comparisons of AVT mRNA in AD and control groups in each preoptic nucleus individually, we found an increase in AVT expression in the parvocellular nucleus of AD-exposed animals $\left[\mathrm{t}_{(14)}=3.75, \mathrm{p}=0.003\right.$; fig. 3,4$]$, but no significant difference between groups in the magnocellular $\left[\mathrm{t}_{(14)}=1.15, \mathrm{p}=0.27\right.$; fig. 3,4$]$ or gigantocellular $\left[\mathrm{t}_{(15)}=1.39, \mathrm{p}=0.19\right.$; fig. 3,4$]$ nuclei.

\section{Discussion}

Previous studies in goldfish demonstrated that intracranial infusion of AVT inhibits social approach towards same-sex conspecifics in males, especially during the breeding season, whereas infusions of a V1a antagonist stimulate social approach behavior [Thompson and Walton, 2004; Thompson et al., 2008b; Walton et al., 2010]; however, the specific social context for these behavioral effects was not entirely clear. In the present study, we demonstrate that a social cue released by both males and females, the pheromone $\mathrm{AD}$, can evoke similar behaviors as AVT administration, but this effect is dependent on the context of the social interaction. We show that AD exposure induces social avoidance in males in response to the presence of another male, but it does not appear to affect the willingness of males to approach the visual stimuli of females. We also show that AD increases AVT gene expression in the preoptic area, which suggests that this social cue may inhibit social approach towards other males by activating the AVT system.

AD is likely an important signal that selectively mediates male-male interactions in goldfish in multisex social groups. During spawning, small groups of males actively chase ovulating females, frequently initiating physical contact with each other ('pushing') in order to maintain a position closest to the female [DeFraipont and Sorensen, 1993; Kobayashi et al., 2002]. Typically, only one or a few males will engage in a spawning act with a female, in which both sexes will release eggs or sperm into vegetation, so social spacing and proximity to the female are important factors in determining mating success. Spawning male goldfish release large quantities of $\mathrm{AD}$ (on average, $15 \times$ more $\mathrm{AD}$ than unstimulated males [Sorensen et al., 2005]), which we have shown acts as a signal to inhibit other males from approaching. In other contexts, 
AD stimulates aggression (pushing) in males housed in the same tank [Poling et al., 2001], indicating that AD can induce different behavioral responses in different social environments, perhaps promoting aggression if a male perceives that he is dominant, or if he cannot retreat, and social withdrawal if he perceives that he is subordinate or if there is an avenue for escape. Importantly, our results show that $\mathrm{AD}$ can suppress the social approach behavior of goldfish males in a way that would likely reduce their ability to compete for females in a scramble competition. Although a relatively understudied phenomenon, the behavioral function of pheromones meant for intermale signaling has also been investigated in other species, such as the red-spotted newt [Notophthalmus vuridescens; Park and Propper, 2001] and leek moth [Acrolepiopsis assectella; Lecomte et al., 1998], in which sexually stimulated males produce pheromones that repel other males competing for mates, thereby increasing the reproductive success of the signaler. Such a system may also benefit the receiver, because pheromone-induced social avoidance allows him to avoid engaging in costly agonistic interactions, perhaps especially when his likelihood of winning is low. As a social signal in goldfish, $\mathrm{AD}$ appears to be very effective in selectively reducing male-male interactions, apparently without influencing the frequency or probability of male-female interactions.

We also demonstrate that $\mathrm{AD}$ exposure increases endogenous AVT in the brain. Fish exposed to AD had increased levels of AVT mRNA in the preoptic area, as measured by in situ hybridization, suggesting that either a greater number of preoptic neurons were producing AVT or that all AVT cells were producing more mRNA in response to the social stimulus. Unfortunately, our method of quantifying AVT expression does not allow us to distinguish between these two possibilities. Taken together with our behavioral study, these results suggest that endogenous AVT release in response to social cues could mediate male-male competition in goldfish and, perhaps more importantly, could regulate more general behavioral processes related to a decrease in sociality. In goldfish, administration of an AVT V1a receptor antagonist blocks the behavioral effects of exogenously administered AVT [Walton et al., 2010] and stimulates social approach in males [Thompson and Walton, 2004; Walton et al., 2010], indicating that endogenous levels are important in predicting the general tendency of males to investigate a conspecific. In the current study, the same social cue that increased AVT in the preoptic area of goldfish males also induced social avoidance in response to another male. We had predicted that $\mathrm{AD}$ might also inhibit approach to- wards females, because they release $\mathrm{AD}$ before they are ready to spawn. However, our results show that while AD decreases time spent approaching male conspecifics, it does not appear to affect behavior towards females, suggesting that the circuit activated by $\mathrm{AD}$ does not modulate approach responses to females. The AVT/AVP system is directly associated with social avoidance in only two other species. In rainbow trout (Onchorhynchus mykiss), intracranial administration of an AVT V1a receptor antagonist increased the latency to withdraw from an agonistic encounter [Backstrom and Winberg, 2009], and in rats an olfactory signal from sick animals promotes avoidance in conspecifics by activating the AVP system in animals that come in contact with the odor [Arakawa et al., 2010]. In some fish species, higher endogenous levels of AVT are correlated with the reduced tendency to display territorial or aggressive behaviors. In the highly territorial beaugregory damselfish (Stegastes leucostictus), the density of AVT-ir fibers in the preoptic area is negatively correlated with the number of times an individual fish approaches and attempts to bite an intruder [Santangelo and Bass, 2010], and in cichlids (Astatotilapia burtoni), adopting a nonterritorial reproductive strategy is associated with higher overall levels of AVT gene expression in the anterior preoptic area [Greenwood et al., 2008]. However, there are several other examples in fish in which increased AVT gene expression is associated with social dominance and increased aggression [Godwin et al., 2000; Aubin-Horth et al., 2007; Greenwood et al., 2008], therefore differences in the patterns of AVT expression in the brain are likely associated with changes in social behavior in a complex way, and may reflect differences in the functional effects of AVT in divergent species and/or differences in AVT neural circuitry underlying behavior.

In fact, Greenwood et al. [2008] recently suggested that AVT in different preoptic cell populations in A. burtoni is associated with the regulation of different behaviors, perhaps through different neural circuitry. In that study, AVT in the parvocellular nucleus is associated with subordinate fleeing behaviors, whereas AVT mRNA expression in the magno- and gigantocellular populations is associated with the expression of territorial behavior. Given our finding that AD induces social withdrawal in response to other males, we expected to find AD-induced AVT specifically in the parvocellular population. Planned post hoc comparisons showed that AVT induction in response to $\mathrm{AD}$ was only significant in the parvocellular portion of the preoptic area, but we did not find a treatment-brain region interaction, so we cannot rule out the 
possibility of AD-induced AVT expression in the magnoand gigantocellular nuclei that could also play a role in regulating withdrawal behavior. Although little is known about the connectivity of individual cell populations in the preoptic area of goldfish, or any fish species, it is clear that some AVT-ir cells from the preoptic area send dense projections to hindbrain autonomic control regions, including the dorsal motor vagus and area postrema [Thompson and Walton, 2009], which play an important role in the effects of AVT on approach behavior in this species by activating a peripheral feedback loop that ultimately promotes social withdrawal [Thompson et al., 2008b]. Thus, we hypothesize that AD could induce social withdrawal in males by activating that circuit. Alternatively, or in addition, AD could activate AVT cells that project to the optic tectum in goldfish [Thompson and Walton, 2009] and thus directly modulate the processing of visual stimuli in ways that lead to decreased social approach. A similar mechanism by which AVT mediates cross-modal sensorimotor integration has been shown in male roughskin newts, in which female sex pheromones enhance the processing of somatosensory stimuli involved in the regulation of courtship clasping [Thompson et al., 2008a].

In summary, AVT plays an important role in modulating social behavior in goldfish by inhibiting approach responses toward conspecifics. Here we have shown that $\mathrm{AD}$, an important chemical signal in the context of repro- duction, induces similar behavioral responses as intracerebral AVT, inducing social avoidance in response to rival males, but not in response to receptive females. Exposure to $\mathrm{AD}$ also increases AVT mRNA expression in the preoptic nucleus of males, suggesting that this communication signal specifically influences male-male interactions by activating a brain circuit, which includes AVT projections to hindbrain vagal efferents, that probably influences social behavior through the integration of peripheral and central effects. Furthermore, AD-induced AVT expression was most robust in the parvocellular population of the preoptic area, suggesting that this brain region in particular may be part of that circuit. We suggest that it would be informative for future studies to investigate the selective AVT responses in separate cell populations of the preoptic nucleus, particularly in different social contexts, in order to better understand how different neuromodulatory pathways shape social approach behavior.

\section{Acknowledgments}

This work was supported by Bowdoin College student fellowships, funded by grants from the National Center for Research Resources (5P20RR016463-12) and the National Institute of General Medical Sciences (8 P20 GM103423-12) from the National Institutes of Health (to A.D.T.K. and E.N.D'A.). Further support was provided by NSF IOS 0849102 to R.R.T.

\section{References}

Albers HE (2012): The regulation of social recognition, social communication and aggression: vasopressin in the social behavior neural network. Horm Behav 61:283-292.

-Arakawa H, Arakawa K, Deak T (2010): Oxytocin and vasopressin in the medial amygdala differentially modulate approach and avoidance behavior toward illness-related social odor. Neuroscience 171:1141-1151.

-Aubin-Horth N, Desjardins JK, Martei YM, Balshine S, Hofmann HA (2007): Masculinized dominant females in a cooperatively breeding species. Mol Ecol 16:1349-1358.

-Backstrom T, Winberg S (2009): Arginine-vasotocin influence on aggressive behavior and dominance in rainbow trout. Physiol Behav 96:470-475

DeFraipont M, Sorensen PW (1993): Exposure to the pheromone 17a, 20b-dihydroxy4-prenen-3-one enhances the behavioral spawning success, sperm production, and sperm motility of male goldfish. Anim Behav 46:245-256.
Dewan AK, Tricas TC (2011): Arginine vasotocin neuronal phenotypes and their relationship to aggressive behavior in the territorial monogamous multiband butterflyfish, Chaetodon multicinctus. Brain Res 1401:74-84.

Gobrogge KL, Liu Y, Jia X, Wang Z (2007): Anterior hypothalamic neural activation and neurochemical associations with aggression in pair-bonded male prairie voles. J Comp Neurol 502:1109-1122.

Godwin J, Sawby R, Warner RR, Crews D, Grober MS (2000): Hypothalamic arginine vasotocin mRNA abundance variation across sexes and with sex change in a coral reef fish. Brain Behav Evol 55:77-84.

-Godwin J, Thompson RR (2012): Nonapeptides and social behavior in fishes. Horm Behav 61: 230-238.

Goodson JL (2008): Nonapeptides and the evolutionary patterning of sociality. Prog Brain Res 170:3-15.
Goodson JL, Bass AH (2001): Social behavior functions and related anatomical characteristics of vasotocin/vasopressin systems in vertebrates. Brain Res Rev 35:246-265.

Goodson JL, Kabelik D, Schrock SE (2009): Dynamic neuromodulation of aggression by vasotocin: influence of social context and social phenotype in territorial songbirds. Biol Lett 5: 554-556.

Goodson JL, Thompson RR (2010): Nonapeptide mechanisms of social cognition, behavior and species-specific social systems. Curr Opin Neurobiol 20:784-794.

-Goodson JL, Wang Y (2006): Valence-sensitive neurons exhibit divergent functional profiles in gregarious and asocial species. Proc Natl Acad Sci USA 103:17013-17017.

-Greenwood AK, Wark AR, Fernald RD, Hofmann HA (2008): Expression of arginine vasotocin in distinct preoptic regions is associated with dominant and subordinate behaviour in an African cichlid fish. Proc R Soc Lond B Biol Sci 275:2393-2402. 
- Ho JM, Murray JH, Demas GE, Goodson JL (2010): Vasopressin cell groups exhibit strongly divergent responses to copulation and male-male interactions in mice. Horm Behav 58:368-377.

Kabelik D, Klatt JD, Kingsbury MA, Goodson JL (2009): Endogenous vasotocin exerts contextdependent behavioral effects in a semi-naturalistic colony environment. Horm Behav 56: 101-107.

Kavaliers M (1989): Day-night rhythms of shoaling behavior in goldfish: opioid and pineal involvement. Physiol Behav 46:167-172.

Kelly AM, Kingsbury MA, Hoffbuhr K, Schrock SE, Waxman B, Kabelik D, Thompson RR, Goodson JL (2011): Vasotocin neurons and septal vla-like receptors potently modulate songbird flocking and responses to novelty. Horm Behav 60:12-21.

Kobayashi M, Sorensen PW, Stacey NE (2002): Hormonal and pheromonal control of spawning behavior in the goldfish. Fish Physiol Biochem 26:71-84.

- Lecomte C, Thibout E, Pierre D, Auger J (1998): Transfer, perception and activity of male pheromone of Acrolepiopsis assectella with special reference to conspecific male sexual inhibition. J Chem Ecol 24:655-671.

Magurran AE, Pitcher TJ (1983): Foraging, timidity and shoal size in minnows and goldfish. Behav Ecol Sociobiol 12:147-152.

- Marler CA, Chu J, Wilczynski W (1995): Arginine vasotocin injection increases probability of calling in cricket frogs, but causes call changes characteristic of less aggressive males. Horm Behav 29:554-570.

Moore FL, Miller LJ (1983): Arginine vasotocin induces sexual behavior of newts by acting on cells in the brain. Peptides 4:97-102.
Park D, Propper CR (2001): Repellent function of male pheromones in the red-spotted newt. J Exp Zool 289:404-408.

Poling KR, Fraser EJ, Sorensen PW (2001): The three steroidal components of the goldfish preovulatory pheromone evoke different behaviors in males. Comp Biochem Physiol B Biochem Mol Biol 129:645-651.

Santangelo N, Bass AH (2010): Individual behavioral and neuronal phenotypes for arginine vasotocin mediated courtship and aggression in a territorial teleost. Brain Behav Evol 75: 282-291.

Scott AP, Sorensen PW (1994): Time course of release of pheromonally active gonadal steroids and their conjugates by ovulatory goldfish. Gen Comp Endocrinol 96:309-323.

- Semsar K, Godwin J (2003): Social influences on the arginine vasotocin system are independent of gonads in a sex-changing fish. J Neurosci 23:4386-4393.

Sorensen PW, Pinillos M, Scott AP (2005): Sexually mature male goldfish release large quantities of androstenedione into the water where it functions as a pheromone. Gen Comp Endocrinol 140:164-175.

-Stacey N, Chojnacki A, Narayanan A, Cole T, Murphy C (2003): Hormonally derived sex pheromones in fish: exogenous cues and signals from gonad to brain. Can J Physiol Pharmacol 81:329-341.

Taborsky M (2001): The evolution of bourgeois, parasitic and cooperative reproductive behaviors in fishes. J Hered 92:100-110.
Thompson RR, Dickinson PS, Rose JD, Dakin KA, Civiello GM, Segerdahl A, Bartlett R (2008a): Pheromones enhance somatosensory processing in newt brains through a vasotocin-dependent mechanism. Proc Biol Sci 275:1685-1693.

Thompson RR, George K, Dempsey J, Walton JC (2004): Visual sex discrimination in goldfish: seasonal, sexual, and androgenic influences. Horm Behav 46:646-654.

Thompson RR, Walton JC (2004): Peptide effects on social behavior: effects of vasotocin and isotocin on social approach behavior in male goldfish (Carassius auratus). Behav Neurosci 118:620-626

Thompson RR, Walton JC (2009): Vasotocin immunoreactivity in goldfish brains: characterizing primitive circuits associated with social regulation. Brain Behav Evol 73:153-164.

- Thompson RR, Walton JC, Bhalla R, George KC, Beth EH (2008b): A primitive social circuit: vasotocin-substance $P$ interactions modulate social behavior through a peripheral feedback mechanism in goldfish. Eur J Neurosci 27: 2285-2293.

Walton JC, Waxman B, Hoffbuhr K, Kennedy M, Beth E, Scangos J, Thompson RR (2010): Behavioral effects of hindbrain vasotocin in goldfish are seasonally variable but not sexually dimorphic. Neuropharmacology 58:126134.

-Wang Z, Ferris CF, DeVries GJ (1994): Role of septal vasopressin innervation in paternal behavior in prairie voles (Microtus ochrogaster). Proc Natl Acad Sci USA 91:400-404.

-Winslow J, Hastings N, Carter CS, Harbaugh C, Insel T (1993): A role for central vasopressin in pair bonding in monogamous prairie voles. Nature 365:545-548. 